\title{
Editorial
}

\section{Editorial for Theme Issue: Applications of KinetiSol Dispersing for Advanced Amorphous Solid Dispersions}

\author{
Dave A. Miller ${ }^{1,2}$
}

Received 6 April 2018; accepted 6 April 2018; published online 8 May 2018

KinetiSol first appeared in the pharmaceutical literature a decade ago as a novel thermal processing technology, adapted from the plastics recycling industry, to produce amorphous solid dispersion (ASD) systems. As a thermal process with a similar narrative of entry into the pharmaceutical space, initial publications focused on differentiating KinetiSol from hot-melt extrusion (HME). These studies identified various KinetiSol advantages over HME, such as the ability to process thermally labile drugs, high melting point drugs, and highly viscous polymers without the need for plasticizers, all stemming from KinetiSol's unique mixing dynamics and brief processing durations.

While initial publications focused on drawing comparisons to and establishing advantages over HME, the commercial position of the process evolved toward that of a complementary technology to HME; an alternative nonsolvent ASD technology to consider when melt extrusion was either not applicable, or failed to produce the desired product. The commercial interest, therefore, put more emphasis on KinetiSol as a competing technology to solvent-based manufacturing approaches. KinetiSol also began to be differentiated as a preferred, or ASD enabling, process when drug properties were not amenable to both spray drying and HME. Finally, with the applicable excipients not being limited to those that are thermally processible, or that share a common solvent with the drug, KinetiSol was found to be highly valuable as a reformulation option to maximize ASD-based drug product performance and value.

In this theme issue, a compilation of five research articles are presented focusing on the development and characterization of advanced ASD systems with specific emphasis on applications of the KinetiSol technology. The aim is to provide a comprehensive perspective on KinetiSol with

Guest Editor: Dave A. Miller

${ }^{1}$ DisperSol Technologies, Georgetown, Texas, USA.

${ }^{2}$ To whom correspondence should be addressed. (e-mail: Dave.miller@dispersoltech.com) particular focus on commercially relevant applications that illustrate the technology's value to Industry. The first article, "Expanding the Application and Formulation Space of Amorphous Solid Dispersions with KinetiSol®: A Review," covers the history and fundamentals of the process, the equipment, and the pharmaceutical literature, as well as commercial examples within the public domain. This article underscores the value of KinetiSol by reviewing its unique principles and highlighting how they translate to differentiated ASD formulations.

The second article, "Improved Vemurafenib Dissolution and Pharmacokinetics as an Amorphous Solid Dispersion Produced by KinetiSol Processing," comparatively evaluates KinetiSol against the micro-precipitated bulk powder (MBP) technology developed by Roche in the commercialization of Zelboraf®. The article demonstrates the ability of KinetiSol to produce a vemurafenib ASD, equivalent in composition to the commercial formulation, without complex solvent-based processing. Surprisingly, it was discovered through non-sink in vitro dissolution testing that the KinetiSol ASD exhibited slower precipitation rates than the equivalent MBP formulation. A pharmacokinetic study in rats also demonstrated a 2.5-fold increase in mean AUC for KinetiSol over MBP. Based on these results, it was hypothesized that the porous structure of the MBP material promoted drug nucleation within the particles, which does not occur within the dense particles of the KinetiSol material. In discussing this result, the paper references recent publications reporting similar findings, suggesting that the structure of ASD particles, and thus the process by which they are formed, may significantly impact performance.

The theme issue then segues into the third article that examines: "Effects of the Preparation Process on the Properties of Amorphous Solid Dispersions." Specifically, this review article analyzes the effects of processing technology on chemical stability, physical stability, and performance, both in vitro and in vivo. The article provides insights, such as solvent-based approaches generally yield ASDs with superior 
chemical stability over thermal methods; however, their physical stability may be compromised by the presence of residual solvent, high surface area, potential nuclei, and molecular relaxation during the secondary drying process. The review also evaluates the various factors (homogeneity of ASD systems, the intermolecular interactions between the drug and polymer, and the morphology of the materials) that impact performance and how those are specifically influenced by the manufacturing technology. The article ultimately concludes by stating that to maximize the properties of an ASD, the manufacturing method must be selected via judicious consideration of the specific properties of the formulation materials.

The forth article, "Generation of a Weakly Acidic Amorphous Solid Dispersion of the Weak Base Ritonavir with Equivalent In-Vitro and In-Vivo Performance to Norvir Tablet," addresses several different issues key to the development of an ASD-based product. Firstly, the article illustrates the importance of polymer selection toward maximizing drug loading without diminishing performance. Secondly, advanced solid-state characterization techniques (solid-state NMR) are employed when traditional methods (modulated DSC) fail to definitively determine the state of drug distribution in the polymer matrix. Finally, the paper examines the appropriate in vitro and in vivo models to employ when comparing formulations of the same drug with opposing
$\mathrm{pH}$ solubility profiles. Ultimately, the study demonstrates the development of a KinetiSol-enabled formulation that is bioequivalent to Norvir tablet at twice the ASD drug loading.

The final article of the issue, "Development of Itraconazole Tablets Containing Viscous KinetiSol ${ }^{\circledR}$ Solid Dispersions: in vitro and in vivo Analysis in Dogs," demonstrates KinetiSol production of itraconazole ASDs with high molecular weight HPMCs and conversion of these ASD intermediates into a tablet dosage form. The article illustrates the supersaturation advantage gained from utilization of highly viscous HPMC and reveals challenges and solutions to developing disintegrating tablets from these ASDs. The paper culminates in a pharmacokinetic study in dogs demonstrating superior mean AUC for one KinetiSol-enabled tablet over the commercial HME-based product, Onmel®.

In summary, this theme issue provides a comprehensive view of the KinetiSol technology from its origin to its current commercial pharmaceutical applications. Highlighted herein are various advantages of KinetiSol ranging from more efficient manufacturing to superior ASD performance stemming from an expanded ASD formulation space and/or unique particle properties. The content of this theme issue illustrates the growth of the KinetiSol technology over the past 10 years into a commercially viable pharmaceutical technology, well differentiated from other ASD processes, and that enables novel, valuable drug products. 